\title{
A SHARP OPERATOR VERSION OF THE BISHOP-PHELPS THEOREM FOR OPERATORS FROM $\ell_{1}$ TO CL-SPACES
}

\author{
LIXIN CHENG, DUANXU DAI, AND YUNBAI DONG
}

(Communicated by Thomas Schlumprecht)

\begin{abstract}
Acosta et al. in 2008 gave a characterization of a Banach space $Y$ (called an approximate hyperplane series property, or AHSP for short) guaranteeing exactly that a quantitative version of the Bishop-Phelps theorem holds for bounded operators from $\ell_{1}$ to the space $Y$. In this note, we give two new examples of spaces having the AHSP: the almost CL-spaces and the class of Banach spaces $Y$ whose dual $Y^{*}$ is uniformly strongly subdifferentiable on some boundary of $Y$. We then calculate the precise parameters associated to almost CL-spaces.
\end{abstract}

\section{INTRODUCTION}

The Bishop-Phelps theorem states that the set of norm-attaining functionals on a Banach space $X$ is dense in the topological dual $X^{*}[2]$. It has been usefully extended in many directions and in the study of optimization. See Phelps' survey [13]. In 1970, B. Bollobás [3] gave a quantitative version of the Bishop-Phelps theorem (known as the Bishop-Phelps-Bollobás theorem). In 1963, J. Lindenstrauss [11] showed that for two Banach spaces $X$ and $Y$, if $X$ is reflexive, then the set $N(X, Y)$ of all norm-attaining operators is always dense in the space $B(X, Y)$ of all bounded linear operators from $X$ to $Y$. He also gave a counterexample showing that it does not hold in general. J. Bourgain [4 proved that $N(X, Y)$ is dense in $B(X, Y)$ if the space $X$ has the Radon-Nikodým property (RNP).

In this paper, the letter $X$ will always be a real Banach space and $X^{*}$ its dual. We denote by $B_{X}\left(S_{X}\right.$, resp.) the closed unit ball (the unit sphere, resp.) of $X$. For a closed convex set $A \subset X$, ext $A$ stands for the set of all extreme points of $A$.

In 2008, Acosta, Aron, García and Maestre [1 introduced two useful notions: the Bishop-Phelps-Bollobás property (BPBP for short) and the approximate hyperplane series property (AHSP for short). Among many other things, they then showed the following insightful result: $\left(\ell_{1}, X\right)$ has the BPBP if and only if $X$ has the AHSP. For two Banach spaces $X, Y$, the pair $(X, Y)$ is said to admit the BPBP provided that that for every $\varepsilon>0$ there exist $\eta(\varepsilon)>0$ and $\beta(\varepsilon)>0$ with $\lim _{t \rightarrow 0} \beta(t)=$ 0 such that for all $T \in S_{B(X, Y)}$, if $x_{0} \in S_{X}$ such that $\left\|T\left(x_{0}\right)\right\|>1-\eta(\varepsilon)$, then

Received by the editors January 21, 2011 and, in revised form, June 17, 2011, June 23, 2011, June 25, 2011 and June 27, 2011.

2010 Mathematics Subject Classification. Primary 47B37, 46B25; Secondary 47A58, 46B20.

Key words and phrases. Norm-attaining operator, Bishop-Phelps theorem, CL-space, Banach space.

The first author was supported by the Natural Science Foundation of China, grant 11771201. 
there exist a point $u_{0} \in S_{X}$ and an operator $S \in S_{B(X, Y)}$ such that

$$
\left\|S u_{0}\right\|=1,\left\|u_{0}-x_{0}\right\|<\beta(\varepsilon) \text { and }\|T-S\|<\varepsilon .
$$

A Banach space $X$ is said to have the AHSP provided that for every $\varepsilon>0$ there exist $0<\delta, \eta<\varepsilon$ such that for every sequence $\left\{x_{k}\right\} \subset S_{X}$ and for every sequence $\left\{t_{k}\right\} \subset \mathbb{R}$ of nonnegative numbers with $\sum_{k=1}^{\infty} t_{k}=1$ satisfying $\left\|\sum_{k=1}^{\infty} t_{k} x_{k}\right\|>1-\eta$, there exist a subset $A \subset \mathbb{N}$, a subset $\left\{z_{k}: k \in A\right\} \subset S_{X}$ and a functional $x^{*} \in S_{X^{*}}$ such that

$$
\text { (1) } \sum_{k \in A} t_{k}>1-\delta ;(2)\left\|z_{k}-x_{k}\right\|<\varepsilon, \forall k \in A \text { and }(3)\left\langle x^{*}, z_{k}\right\rangle=1, \forall k \in A \text {. }
$$

In this note, we show first that both real almost $C L$-spaces and real Banach spaces $Y$ whose dual is uniformly strongly subdifferentiable on some boundary of $Y$ satisfy the AHSP; hence for these spaces the pair $\left(\ell_{1}, Y\right)$ satisfies the BPBP. Indeed, if $Y$ is an almost CL-space, $\varepsilon>0$, and $x_{0} \in S_{\ell_{1}} T \in S_{\left(\ell_{1}, Y\right)}$ satisfies $\left\|T x_{0}\right\| \geq 1-\varepsilon^{2}$, then there exists an operator $S \in S_{\left(\ell_{1}, Y\right)}$ and an element $y_{0} \in \ell_{1}$ satisfying

$$
\left\|S y_{0}\right\|=1, \quad\|S-T\| \leq \varepsilon, \text { and }\left\|y_{0}-x_{0}\right\|<2 \varepsilon .
$$

\section{The MAIN RESUlts}

The notion of a CL-space was first introduced by R. Fullerton [8] in 1960, and a generalized notion of a CL-space (i.e., an almost CL-space) was introduced by A. Lima [10] in 1978. A Banach space is called a (an almost) CL-space provided its closed unit ball is the (closed) absolutely convex hull of each maximal convex set of the unit sphere. For example, for any nonempty set $\Gamma$ the real Banach spaces $c_{0}(\Gamma), \ell_{1}(\Gamma), \ell_{\infty}(\Gamma)$, and for any measure $\mu$ the real spaces $L_{1}(\mu), L_{\infty}(\mu)$, and $C(K)$ for any compact space $K$ are CL-spaces (see, for instance, [12]). Assume that $X$ is a Banach space and that $C$ is a maximal convex set of the unit sphere $S_{X}$. Then there exists an extreme point $x^{*}$ of the dual unit ball $B_{X^{*}}$ such that $C=\left\{x \in B_{X}:\left\langle x^{*}, x\right\rangle=1\right\}$ (see, for instance, [5]).

Theorem 2.1. Assume that $X$ is an almost CL-space and $0<\varepsilon<1$. Then for every sequence $\left\{x_{n}\right\} \subset B_{X}$ and every convex series $\sum_{n} t_{n}$ satisfying

$$
\left\|\sum_{n=1}^{\infty} t_{n} x_{n}\right\|>1-\varepsilon^{2},
$$

there exist $A \subset \mathbb{N},\left\{z_{k}: k \in A\right\} \subset S_{X}$ and $x^{*} \in \operatorname{ext}\left(B_{X^{*}}\right)$ such that
(1) $\sum_{k \in A} t_{k}>1-\varepsilon$;
(2) $\left\|z_{k}-x_{k}\right\|<\varepsilon, \forall k \in A$ and
(3) $\left\langle x^{*}, z_{k}\right\rangle=1, \forall k \in A$.

As a consequence, $X$ has the AHSP.

Proof. Suppose that the sequence $\left\{x_{n}\right\} \subset B_{X}$ and the convex series $\sum_{n} t_{n}$ satisfies the assumption; that is,

$$
\left\|\sum_{n=1}^{\infty} t_{n} x_{n}\right\|>1-\varepsilon^{2} .
$$

We can choose $0<\eta<\varepsilon$ small enough such that

$$
\left\|\sum_{n=1}^{\infty} t_{n} x_{n}\right\|>1-(\varepsilon-\eta)^{2} .
$$


In view of the Hahn-Banach theorem there is $x^{*} \in S_{X^{*}}$ such that

$$
\left\langle x^{*}, \sum_{n=1}^{\infty} t_{n} x_{n}\right\rangle=\left\|\sum_{n=1}^{\infty} t_{n} x_{n}\right\|>1-(\varepsilon-\eta)^{2} .
$$

By Zorn's lemma, the (nonempty) convex subset

$$
C:=\left\{x \in B_{X}:\left\langle x^{*}, x\right\rangle=1\right\}
$$

is contained in a maximal convex subset of the unit sphere $S_{X}$. Hence, by changing the functional $x^{*}$ by an appropriate extreme point of $B_{X^{*}}$ (if needed), we can assume that $C$ is a maximal convex subset of $S_{X}$.

We take $A:=\left\{k \in \mathbb{N}:\left\langle x^{*}, x_{k}\right\rangle>1-\varepsilon+\eta\right\}$. It is clear that

$$
\begin{gathered}
1-(\varepsilon-\eta)^{2} \leq\left\langle x^{*}, \sum_{n=1}^{\infty} t_{n} x_{n}\right\rangle \\
\leq \sum_{n=1}^{\infty} t_{n}+(1-\varepsilon+\eta)\left(1-\sum_{n \in A} t_{n}\right) .
\end{gathered}
$$

Hence

$$
1-\varepsilon<1-(\varepsilon-\eta) \leq \sum_{n \in A} t_{n}
$$

By using now the assumption that $X$ is an almost CL-space, $B_{X}=\overline{\mathrm{co}}(C \cup-C)$, and so there are sequences $\left\{z_{k}\right\} \subset C,\left\{y_{k}\right\} \subset-C$ and $\left\{\alpha_{k}\right\} \subset[0,1]$ satisfying that

$$
\| x_{k}-\left(\alpha_{k} z_{k}+\left(1-\alpha_{k}\right) y_{k} \|<\frac{\eta}{2}, \forall k \in \mathbb{N} .\right.
$$

For every $k \in A$ we have

$$
\begin{gathered}
1-(\varepsilon-\eta) \leq\left\langle x^{*}, x_{k}\right\rangle \leq \frac{\eta}{2}+\left\langle x^{*}, \alpha_{k} z_{k}+\left(1-\alpha_{k}\right) y_{k}\right\rangle \\
=\frac{\eta}{2}+2 \alpha_{k}-1 .
\end{gathered}
$$

As a consequence,

$$
2\left(1-\alpha_{k}\right) \leq \frac{\eta}{2}+\varepsilon-\eta, \forall k \in A .
$$

We deduce for every $k \in A$ that

$$
\begin{gathered}
\left\|x_{k}-z_{k}\right\| \leq\left\|x_{k}-\left(\alpha_{k} z_{k}+\left(1-\alpha_{k}\right) y_{k}\right)\right\|+\left\|\left(\alpha_{k} z_{k}+\left(1-\alpha_{k}\right) y_{k}\right)-z_{k}\right\| \\
<\frac{\eta}{2}+2\left(1-\alpha_{k}\right) \leq \frac{\eta}{2}+\frac{\eta}{2}+\varepsilon-\eta=\varepsilon
\end{gathered}
$$

Hence the statement is proved.

In the following, we shall show that every pair $\left(\ell_{1}, Y\right)$ has the BPBP (or, equivalently, $Y$ has the AHSP) if $Y$ is in the class of Banach spaces whose dual norm is uniformly strongly subdifferentiable (USSD for short) on some boundary of $Y$. The following notions can be found in [6, 7] and [9]. A convex function $f$ defined on a Banach space $X$ is said to be strongly subdifferentiable (SSD) at $x \in X$ if the one-side $\operatorname{limit}_{\lim _{t \rightarrow 0+}} \frac{f(x+t h)-f(x)}{t}$ exists uniformly for $h \in S_{X}$, and it is called uniformly strongly subdifferentiable (USSD) on a subset $A$ of $X$ if the limit above exists uniformly for $x \in A$ and $h \in S_{X}$. For a nonempty closed bounded convex set $C$ in a Banach space $X$, the mapping $J_{C}: X^{*} \rightarrow 2^{C}$ is defined for $x^{*} \in X^{*}$ by $J_{C}\left(x^{*}\right)=\left\{x \in C:\left\langle x^{*}, x\right\rangle=\sup _{z \in C}\left\langle x^{*}, z\right\rangle\right\}$. If, in particular, $C=B_{X}$, then we simply write $J_{X}$ instead of $J_{B_{X}}$. Note that in this case 
$J_{X}\left(x^{*}\right)=\left\{x \in S_{X}:\left\langle x^{*}, x\right\rangle=\left\|x^{*}\right\|\right\}$. A subset $B \subset B_{X^{*}}$ is said to be a boundary (also called a James' boundary) of a Banach space $X$ if for each $x \in X$ there is an $x^{*} \in B$ such that $\left\langle x^{*}, x\right\rangle=\|x\|$; that is, a boundary is an exact norming set of $X$ contained in $B_{X^{*}}$. Clearly, $J_{X^{*}}\left(S_{X}\right)$ is a boundary of $X$.

The following proposition is a consequence of Proposition 2.2 in G. Godefroy 9 for strong subdifferentiability (SSD) of the norm. C. Franchetti and R. Payá 7 ] have also shown that $(i) \Leftrightarrow(i i)$.

Proposition 2.2. Suppose that $X$ is a Banach space. Then the following statements are equivalent:

(i) the dual norm of $X^{*}$ is $S S D$ at $x^{*} \in X^{*}$;

(ii) the map $J_{X^{* *}}$ is norm-to-norm upper semi-continuous at $X^{*}$. That is, for every $\epsilon>0$, there exists $\delta>0$ such that $J_{X^{* *}}\left(y^{*}\right) \subseteq J_{X^{* *}}\left(x^{*}\right)+\epsilon B_{X^{* *}}$ whenever $y^{*} \in S_{Y^{*}}$, satisfying $\left\|y^{*}-x^{*}\right\|<\delta$; that

(iii) the set $J_{X}\left(x^{*}\right)$ is nonempty, and for every $\epsilon>0$, there exists $\delta>0$ such

$$
x \in B_{X},\left\langle x^{*}, x\right\rangle>\left\|x^{*}\right\|-\delta \Longrightarrow d\left(x, J_{X}\left(x^{*}\right)\right)<\varepsilon ;
$$

(iv) for every $\epsilon>0$, there exists $\delta>0$ such that

$$
x^{* *} \in B_{X^{* *}},\left\langle x^{* *}, x^{*}\right\rangle>\left\|x^{*}\right\|-\delta \Longrightarrow d\left(x^{* *}, J_{X^{* *}}\left(x^{*}\right)\right)<\epsilon .
$$

Analogously, we have

Proposition 2.3. Suppose that $X$ is a Banach space and $B \subset S_{X^{*}}$ is a boundary of $X$. Then the following statements are equivalent:

(i) the dual norm of $X^{*}$ is USSD on the boundary B;

(ii) the map $J_{X^{* *}}$ is norm-to-norm uniformly upper semi-continuous on $B$. That is, for every $\epsilon>0$, there exists $\delta>0$ such that $J_{X^{* *}}\left(y^{*}\right) \subseteq J_{X^{* *}}\left(x^{*}\right)+\epsilon B_{X^{* *}}$ whenever $x^{*} \in B$ and $y^{*} \in S_{Y^{*}}$, satisfying $\left\|y^{*}-x^{*}\right\|<\delta$.

(iii) for every $\epsilon>0$, there exists $\delta>0$ such that $d\left(x, J_{X}\left(x^{*}\right)\right)<\epsilon$ whenever $x \in B_{X}$ and $x^{*} \in B$, satisfying $\left\langle x^{*}, x\right\rangle>1-\delta$.

Note Proposition 2.3 (iii) entails that the Banach space $X$ has the AHSP. As a consequence of [1], we have

Corollary 2.4. Suppose that $Y$ is a Banach space and that the dual norm of $Y^{*}$ is USSD on a boundary $B$ of $Y$. Then the pair $\left(\ell_{1}, Y\right)$ admits the BPBP.

Acosta, Aron, García and Maestre [1] have shown that if $X$ is uniformly convex, then $X$ has the AHSP. Since $X$ is uniformly convex if and only if $X^{*}$ is uniformly smooth, we have

Corollary 2.5 (Acosta-Aron-García-Maestre). Suppose that $Y$ is a uniformly convex Banach space. Then the pair $\left(\ell_{1}, Y\right)$ has the BPBP.

Remark 2.6. If $X$ is an almost $C L$-space, then for every $\epsilon>0$, we have $d\left(x, J_{X}\left(x^{*}\right)\right)$ $<\epsilon$ whenever $x \in B_{X}$ and $x^{*} \in B$ satisfying $\left\langle x^{*}, x\right\rangle>1-\epsilon$, where $B$ denotes the boundary mex $\left(B_{X^{*}}\right) \subset \operatorname{ext}\left(B_{X^{*}}\right)$ of $X$ consisting of those extreme points $x^{*}$ satisfying the fact that $C \equiv\left\{x \in S_{X}:\left\langle x^{*}, x\right\rangle=1\right\}$ is a maximal convex set of $S_{X}$. Therefore, every almost $C L$-space admits the property that the dual norm is USSD on the boundary mex $\left(B_{X^{*}}\right)$ of $X$.

We have proven that almost CL-spaces have the AHSP (Theorem 2.1), so in view of Theorem 4.1 of [1], for an almost CL-space $Y$, the pair $\left(\ell_{1}, Y\right)$ has the BPBP. 
Even so, for the sake of completeness we will give a direct proof of the last fact and give functions $\eta$ and $\beta$ satisfying the definition of the BPBP in this case.

Theorem 2.7. Suppose that $Y$ is an almost $C L$-space, $\varepsilon>0$, and that $x_{0} \in \ell_{1}$ with $\left\|x_{0}\right\|=1$. Let $T \in B\left(\ell_{1}, Y\right)$ with $\|T\|=1$ such that $\left\|T\left(x_{0}\right)\right\| \geq 1-\varepsilon^{2}$. Then there exist a point $y_{0} \in S_{\ell_{1}}$ and a norm-attaining operator $S \in S_{B\left(\ell_{1}, Y\right)}$ such that

$$
\text { (1) }\left\|S\left(y_{0}\right)\right\|=1 ; \quad(2)\|S-T\| \leq \varepsilon \text { and (3) }\left\|y_{0}-x_{0}\right\|<2 \varepsilon .
$$

Proof. Let $x_{0} \in \ell_{1}$ with $\left\|x_{0}\right\|=\sum_{n=1}^{\infty}\left|x_{0}(n)\right|=1$ satisfy $\left\|T x_{0}\right\|>1-\epsilon^{2}$. Let $C$ be a maximal convex set of $S_{Y}$ containing $v_{0} \equiv T\left(x_{0}\right) /\left\|T\left(x_{0}\right)\right\|$. Then there exists $x^{*} \in \operatorname{ext}\left(B_{Y^{*}}\right)$ such that $C=\left\{u \in S_{Y}:\left\langle x^{*}, u\right\rangle=1\right\}$. Therefore, $\left\langle x^{*}, T x_{0}\right\rangle=$ $\left\|T x_{0}\right\|$.

Let $\left\{e_{n}\right\}_{n=1}^{\infty}$ be the canonical basis of $\ell_{1}$. Applying Theorem 2.1 to the nonnegative numbers $t_{n}=\left|x_{0}(n)\right|$ and the vectors $y_{n}=T\left(\operatorname{sgn} x_{0}(n) e_{n}\right)$ (for all $\left.n \in \mathbb{N}\right)$, there exist a set $A \subset \mathbb{N}$ and a set $\left\{z_{n}: n \in A\right\} \subset Y$ with $\left\|z_{n}\right\|=1$ for all $n \in A$ such that

$$
\sum_{n \in A}\left|x_{0}(n)\right|>1-\epsilon \text { and }\left\|z_{n}-y_{n}\right\|<\epsilon,\left\langle x^{*}, z_{n}\right\rangle=1
$$

and

$$
\left\|\sum_{n \in A}\left|x_{0}(n)\right| z_{n}\right\|=\sum_{n \in A}\left|x_{0}(n)\right| .
$$

We define $S: \ell_{1} \rightarrow Y$ for $x \in \ell_{1}$ by $S(x)=\sum_{n=1}^{\infty} x(n) u_{n}$, where

$$
u_{n}= \begin{cases}\operatorname{sgn} x_{0}(n) z_{n}, & \text { if } n \in A ; \\ T\left(e_{n}\right), & \text { if } n \notin A .\end{cases}
$$

It is clear that $\|S\|=1$. Let $y_{0}=\sum_{n \in A} x_{0}(n) e_{n} / \sum_{n \in A}\left|x_{0}(n)\right|$. Then

$$
\begin{aligned}
&\left\|y_{0}\right\|=1,\left\|S\left(y_{0}\right)\right\|=\left\|\sum_{n \in A} x_{0}(n) u_{n}\right\| / \sum_{n \in A}\left|x_{0}(n)\right|=\left\|\sum_{n \in A}\left|x_{0}(n)\right| z_{n}\right\| / \sum_{n \in A}\left|x_{0}(n)\right|=1, \\
&\left\|y_{0}-x_{0}\right\| \leq\left\|y_{0}-\sum_{n \in A} x_{0}(n) e_{n}\right\|+\left\|\sum_{n \in A} x_{0}(n) e_{n}-x_{0}\right\| \\
&=\left(1-\left\|\sum_{n \in A} x_{0}(n) e_{n}\right\|\right)+\left\|\sum_{n \in A} x_{0}(n) e_{n}-x_{0}\right\| \\
&=\sum_{n \notin A}\left|x_{0}(n)\right|+\left\|\sum_{x \notin A} x_{0}(n) e_{n}\right\|<\varepsilon+\varepsilon=2 \varepsilon,
\end{aligned}
$$

and

$$
\|S-T\|=\sup _{n}\left\|(S-T)\left(e_{n}\right)\right\|=\sup _{n \in A}\left\|z_{n}-y_{n}\right\| \leq \epsilon .
$$

\section{ACKNOWLEDGEMENTS}

The authors would like to express their deep gratitude to the referee for very helpful comments, suggestions and corrections. In fact, because of the referee's suggestions, Theorem 2.1 is a combination of a lemma and a theorem of a previous version of this note, and its proof presented here was also suggested by the referee. 


\section{REFERENCES}

[1] M. D. Acosta, R. M. Aron, D. García, M. Maestre, The Bishop-Phelps-Bollobás theorem for operators, J. Funct. Anal. 254 (2008), no. 11, 2780-2799. MR2414220 (2009c:46016)

[2] E. Bishop and R. R. Phelps, A proof that every Banach space is subreflexive, Bull. Amer. Math. Soc. 67 (1961), 97-98. MR0123174 (23:A503)

[3] B. Bollobás, An extension to the theorem of Bishop and Phelps, Bull. London Math. Soc. 2 (1970), 181-182. MR0267380 (42:2282)

[4] J. Bourgain, On dentability and the Bishop-Phelps property, Israel J. Math. 28 (1977), 265271. MR0482076 (58:2164)

[5] L. Cheng and M. Li, Extreme points, exposed points, differentiability points in CL-spaces, Proc. Amer. Math. Soc. 136 (2008), no. 7, 2445-2451. MR2390512 (2009a:46021)

[6] R. Deville, G. Godefroy, V. Zizler, Smoothness and renormings in Banach spaces. Pitman Monographs and Surveys in Pure and Applied Mathematics, 64. Longman Scientific \& Technical, Harlow; copublished in the United States with John Wiley \& Sons, Inc., New York, 1993. MR 1211634 (94d:46012)

[7] C. Franchetti and R. Payá, Banach spaces with strongly differentiable norm, Boll. U.M.I. 7 (1993), 45-70. MR1216708 (94d:46015)

[8] R. E. Fullerton, Geometrical characterizations of certain function spaces, in Proc. Internat. Sympos. Linear Spaces (Jerusalem, 1960), pp. 227-236, Jerusalem Academic Press, Jerusalem; Pergamon Press, Oxford, 1961. MR0132998 (24:A2834)

[9] G. Godefroy, V. Indumathi, F. Lust-Piquard, Strong subdifferentiability of convex functionals and proximinality, J. Approx. Theory 116 (2002), 397-415. MR1911087 (2003d:41034)

[10] Å. Lima, Intersection properties of balls and subspaces in Banach spaces, Trans. Amer. Math. Soc. 227 (1977), 1-62. MR0430747 (55:3752)

[11] J. Lindenstrauss, On operators which attain their norm, Israel J. Math. 1 (1963), 139-148. MR.0160094 (28:3308)

[12] M. Martin and R. Payá, On CL-spaces and almost CL-spaces, Ark. Mat. 42 (2004), 107-118. MR2056547(2005e:46019)

[13] R. R. Phelps, The Bishop-Phelps theorem, Ten mathematical essays on approximation in analysis and topology, 235-244, edited by J. Ferrera, J. López-Gómez and F. R. Ruiz del Portal, Elsevier B. V., Amsterdam, 2005. MR2162983 (2006d:46015)

School of Mathematical Sciences, Xiamen University, Xiamen, 361005, People's Republic of China

E-mail address: 1xcheng@xmu.edu.cn

School of Mathematical Sciences, Xiamen University, Xiamen, 361005, People's RePublic OF CHINA

E-mail address: dduanxu@163.com

School of Mathematical Sciences, Xiamen University, Xiamen, 361005, People's Republic of China

E-mail address: Baiyunmu301@126.com 